\title{
Which placebo to cure depression? A thought-provoking network meta-analysis
}

\author{
Florian Naudet ${ }^{1,2,3^{*}}$, Bruno Millet ${ }^{2,3}$, Philippe Charlier ${ }^{4,5}$, Jean Michel Reymann ${ }^{6,7}$, Anne Solène Maria ${ }^{1,8}$ \\ and Bruno Falissard ${ }^{1,8,9}$
}

\begin{abstract}
Background: Antidepressants are often considered to be mere placebos despite the fact that meta-analyses are able to rank them. It follows that it should also be possible to rank different placebos, which are all made of sucrose. To explore this issue, which is rather more epistemological than clinical, we designed an unusual meta-analysis to investigate whether the effects of placebo in one situation are different from the effects of placebo in another situation.

Methods: Published and unpublished studies were searched for by three reviewers on Medline, the Cochrane Library, Embase, clinicaltrials.gov, Current Controlled Trial, in bibliographies, and by mailing key organizations. The following studies in first-line treatment for major depressive disorder were considered to construct an "evidence network": 1) randomized controlled trials (RCTs) versus placebo on fluoxetine, venlafaxine and 2) fluoxetine versus venlafaxine head-to-head RCTs.

Two network meta-analyses were run to indirectly compare response and remission rates among three different placebos: 1) fluoxetine placebo, 2) venlafaxine placebo, and 3) venlafaxine/fluoxetine placebo (that is, placebo compared to both venlafaxine and fluoxetine). Publication biases were assessed using funnel plots and statistically tested.
\end{abstract}

Results: The three placebos were not significantly different in terms of response or remission. The antidepressant agents were significantly more efficacious than the placebos, and venlafaxine was more efficacious than fluoxetine. The funnel plots, however, showed a major publication bias.

Conclusion: The presence of significant levels of publication bias indicates that we cannot even be certain of the conclusion that sucrose equals sucrose in trials of major depressive disorder. This result should remind clinicians to step back to take a more objective view when interpreting a scientific result. It is of crucial importance for their practice, far more so than ranking antidepressant efficacy.

Keywords: Antidepressants, Placebo, Major depressive disorder, Meta-analysis, Publication bias

\section{Background}

The history of medicine is closely linked to the history of placebos. Pre-scientific medicine was based on many bizarre and ineffective medical interventions and on the belief that such treatments were effective [1]. Placebo was used for the first time as a control in 1784 to debunk the healing claims of mesmerism [2], and it

\footnotetext{
*Correspondence: floriannaudet@gmail.com

${ }^{1}$ INSERM U669, Maison de Solenn, 97 Boulevard de Port Royal, 75679 Paris Cedex 14, France

¿Université de Rennes 1, EA-4712 Behavior and Basal Ganglia Unit, Rennes, France

Full list of author information is available at the end of the article
}

became a standard control in experimental procedures in the second half of the $20^{\text {th }}$ century. Randomized controlled trials (RCTs) against placebo have enabled major progress in modern medicine. Nevertheless, these studies have limitations in terms of external validity and even internal validity, and antidepressant literature on major depressive disorder is a striking example of these limitations: some practitioners and researchers [3] consider that most of the antidepressant efficacy reflects simply the placebo effect, especially for depression in patients with mild or moderate symptoms $[4,5]$. However, many patients are satisfied with these treatments, many

\section{Biomed Central}


clinicians trust them and use them, and a large part of discussions during medical staff meetings is devoted to the choice of the right sort of antidepressant drug [6]. Recently, Cipriani et al. in a multiple-treatment metaanalysis, ranked 12 new-generation antidepressants [7] to address this question.

This state-of-the-art raises a fundamental question: if much of the effect of antidepressants is attributable to the placebo effect and if it is possible to rank antidepressants, then it should also be possible to rank different placebos, which are all made of sucrose. In a more global perspective, it questions whether or not we can be certain about anything in psychiatry (or, indeed, in medicine), and, in particular, whether the evidence that we usually rely on provides us with a reasonable degree of certainty about the nature and effectiveness of our practices. We set about investigating this question, which is rather more epistemological than clinical, by investigating whether the effects of placebo in one situation are different from the effects of placebo in another situation. We thus designed an unusual meta-analysis on aggregated data which allows us to examine the apparently incontrovertible fact that sucrose equals sucrose by comparing the placebos of two famous antidepressant blockbusters: 1) fluoxetine, one of the first selective serotonin reuptake inhibitors available on the market, which has become a reference drug, and 2) venlafaxine, a serotoninnorepinephrine reuptake inhibitor.

\section{Methods}

\section{Eligibility criteria}

\section{Types of participants}

We reviewed studies involving adults (age 18 and over) with a diagnosis of major depressive episode (DSM IV, DSM IV-R, DSM III, DSM III-R, ICD 10, Feighner criteria, Research Diagnostic Criteria). Studies involving patients with other psychiatric or medical comorbidity were considered only when it was not an explicit inclusion criterion for the study. Studies involving patients with a diagnosis of anxious depression were also considered.

Studies involving more than $20 \%$ subjects with bipolar disorder were excluded, as were studies exclusively involving patients with seasonal affective disorder, postpartum depression, postmenopausal depression, atypical depression, dysthymia and studies involving elderly patients.

\section{Types of intervention(s)}

Our primary aim was to compare placebo arms. We focused our attention on three different placebos: 1) fluoxetine placebo (FLUp, where placebo was compared to fluoxetine), 2) venlafaxine placebo (VENLAFp, where placebo was compared to venlafaxine), and 3 ) venlafaxine/fluoxetine placebo (FLU/VENLAFp, where placebo was compared to both venlafaxine and fluoxetine), which were obviously compared with the corresponding antidepressants in oral mono-therapy in major depressive disorder first line acute treatment.

\section{Types of outcomes}

Response was chosen as the primary outcome. Remission was chosen as a secondary outcome. These outcomes are the most consistently reported estimates of acute-treatment efficacy. Response was defined as the proportion of patients who had a reduction of at least $50 \%$ from the baseline score on the Hamilton Depression Rating Scale (HDRS) [8] or the Montgomery-Åsberg Depression Rating Scale (MADRS) [9]. Remission was defined as the proportion of patients who had a HDRS score $\leq 7$ or a MADRS score $\leq 12$.

When trials reported results from both rating scales, we extracted data from the scale considered in the study as the primary outcome.

\section{Types of studies}

In this review were included 1) randomized controlled trials of fluoxetine or venlafaxine against placebo and 2) head-to-head trials of fluoxetine versus venlafaxine with or without placebo control. All studies were conducted from January 1989 to July 2009. Only study reports in English, French and Spanish language were considered.

\section{Search strategy}

We used the search strategy from an earlier paper [10] on venlafaxine and fluoxetine to conduct this metaanalysis on aggregated data.

Eligible studies were identified from PubMed/Medline, the Cochrane library and Embase, including congress abstracts. A three-step search was used for each component of this review. In a first step, an initial search on Medline was carried out in order to refresh optimal search terms and include possible changes in the databases. The search terms used were double-checked before starting the main search. In a second step, all keywords identified were used to search all the abovementioned databases. A third search was undertaken by searching the reference lists of articles identified. Initial keywords used were: "Depressive Disorder NOT Depression, Postpartum NOT Seasonal Affective Disorder"; "Antidepressive Agents"; "Fluoxetine"; "Venlafaxine". In addition, manual searches of articles were performed in previous meta-analyses.

Unpublished studies were searched for by communication with key organizations such as the Food and Drug Administration (FDA) and the European Medicines Agency (EMEA), and key researchers in the area. A search on clinicaltrials.gov and Current Controlled Trial was performed. 
Authors of abstracts or meta-analysis were contacted for further information and were asked for references of the studies when needed. If no response was obtained to a first solicitation, they were then contacted a second time.

\section{Study selection}

Eligibility assessment was performed independently in a blinded standardized manner by two reviewers (NF and MAS). Disagreements were resolved by consensus or in consultation with a third reviewer (FB).

A comparison across the studies, checking author names, treatment comparisons, sample sizes and outcomes was performed to avoid duplicates and compilations of data from several reports of the same study.

\section{Assessment of methodological quality}

Each paper was then assessed for methodological quality prior to inclusion in the review using an appropriate standardized critical appraisal instrument from the Joanna Briggs Institute [11].

\section{Data collection}

A data extraction sheet based on the Cochrane Handbook for Systematic Reviews of Interventions' guidelines (Version 5.0.2, updated September 2009) [12] was used. One review author (NF) extracted these data from the studies included.

\section{Data analysis}

The dichotomous outcomes used here are considered as robust outcome measures of treatment efficacy [7]. When these outcomes were not reported, the studies were excluded, and no imputation method was used. Responder and remitter data were extracted as the original study investigators analyzed the data, mainly using the LOCF (Last Observation Carried Forward) method.

\section{Head-to-head direct evidence}

We used visual inspection of the forest plots and the $Q$ statistic [13] to investigate the possibility of statistical heterogeneity. In the absence of heterogeneity we performed pair-wise meta-analyses by synthesizing studies that compared the same interventions with a fixedeffects model (Mantel-Haenszel); in case of possible heterogeneity, we performed pair-wise meta-analysis with a random effect model (DerSimonian and Laird).

\section{Network meta-analyses to enable indirect comparisons}

In a second step, to compare indirectly the different placebos, and following recommendations by Glenny et al. [14], two network meta-analyses [15,16] were run. The dependent variables were 1) response and 2) remission; the treatment was considered as the explanatory variables (fluoxetine, venlafaxine, FLUp, VENLAFp and
FLU/VENLAFp). Both fixed effect and random effect approaches were performed for each network metaanalysis; final models were selected by comparing a model fit criterion (Akaike's Information Criterion (AIC)). Results of these meta-analyses are the odds ratio (OR) between treatments with their 95\% confidence interval and the statistical significance level of the comparison.

\section{Risk of bias across studies}

Publication bias was investigated graphically using funnel plots for each fixed effect meta-analysis. Funnel plot asymmetry was tested using the rank correlation test when there were at least 10 studies [17].

Analyses were performed using $\mathrm{R}$ [18] with libraries meta [19], rmeta [20] and lme4 [21] (lmer function, family = binomial). Results are presented according to PRISMA (Preferred Reporting Items for Systematic Reviews and Meta-Analyses) statements [22].

\section{Results}

\section{Study selection}

The search of Medline, Cochrane and Embase databases provided a total of 11,051 citations. An additional 66 studies were identified by manual search. After adjusting for duplicates, 4,615 remained. Of these, 4,063 studies were discarded because, after review of the abstracts, it appeared that these papers did not meet the criteria. A total of 114 studies were excluded because of the language (among which 97 were in Chinese). Of 33 unpublished relevant studies identified, only 3 were made available by pharmaceutical firms. Thirty-one studies were included in the quantitative review. A flow-chart detailing the study selection process is given in Figure 1.

\section{Study characteristics and risk of bias within studies}

A summary of the methodologies of these 31 studies is given in Table 1. The quality of the different studies is presented as a table in a web appendix (Additional file 1: Table S1). The "response" analysis involved 29 RCTs including 7,459 participants and the "remission" analysis involved 20 RCTs including 5,096 participants.

\section{Results from individual studies and synthesis of results Head-to-head direct evidence}

Using the Q statistic, no significant heterogeneity was detected for fluoxetine versus placebo, venlafaxine versus placebo or for fluoxetine versus venlafaxine in the response and remission meta-analyses. Nevertheless, as visual inspection of the forest plot suggested that heterogeneity could not be totally excluded, and as heterogeneity tests are often under-powered [23], we ran pair-wise fixed effects and random effects models which found the same results. 


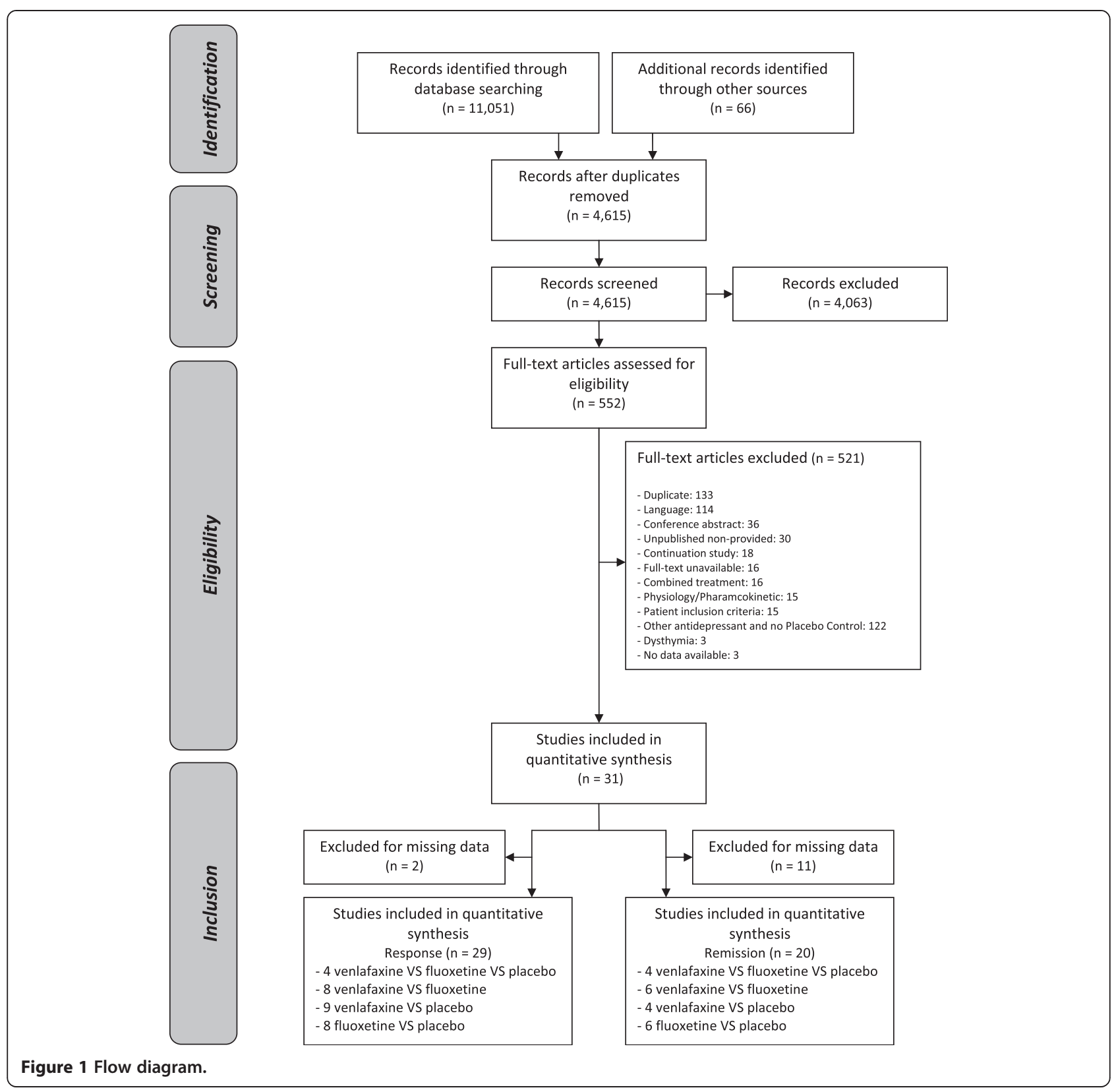

Results of the head-to-head direct meta-analysis using a fixed-effects model are presented in Figure 2 and in the web-appendix (Additional file 1: Table S2) and results of random-effects model are presented in the web appendix (Additional file 1: Table S2 and Additional file 1: Figure S1): regarding treatment response, placebo appeared less effective than both fluoxetine and venlafaxine. Fluoxetine appeared less effective than venlafaxine.

\section{Network meta-analyses to enable indirect comparisons}

Figure 3 shows all the available evidence for the metaanalysis concerning the response. Concerning the two network meta-analyses (response and remission), AIC (presented in the e-appendix, Additional file 1: Table S3) was in favor of the selection of the fixed effect network meta-analysis (which fits the data better).

Table 2 summarizes the results of these two fixedeffect network meta-analyses concerning response and remission. The three placebos were not significantly different in terms of response $(P=0.33$ for FLU/VENLAFp versus VENLAFp, $P=0.50$ for FLU/VENLAFp versus FLUp and $P=0.85$ for VENLAFp versus FLUp) or remission $(P=0.88$ for FLU/VENLAFp versus VENLAFp, $P=0.66$ for FLU/VENLAFp versus FLUp and $P=0.76$ for VENLAFp versus FLUp). Antidepressant agents were significantly more efficacious than placebos and venlafaxine 
Table 1 Summary of study methodology

\begin{tabular}{|c|c|c|c|c|c|}
\hline & V vs $F$ vs $P(n=4)$ & V vs $F(n=8)$ & V vs $P(n=10)$ & $F$ vs $P(n=9)$ & Total $(n=31)$ \\
\hline Year & $\begin{array}{l}(1999,2003, \\
2004,2009)\end{array}$ & $\begin{array}{c}(1994,1997,1998 \\
2000,2007)\end{array}$ & $\begin{array}{c}(1993,1996,1997, \\
1998,2004)\end{array}$ & $\begin{array}{c}(1991,1998,2002 \\
2004,2005)\end{array}$ & $\begin{array}{c}(1991,1997,1999 \\
2003,2009)\end{array}$ \\
\hline Anxious depression (No) & $3(75 \%)$ & $7(88 \%)$ & $10(100 \%)$ & $8(89 \%)$ & $28(90 \%)$ \\
\hline Study duration (weeks) & $(6,6,8,12)$ & $(6,8,9,12,12)$ & $(4,6,8,11,13)$ & $(4,6,8,12,13)$ & $(4,6,8,12,13)$ \\
\hline Number of follow-up visits & $(6,7,8,8)$ & $(6,6,7,8,9)(N A=1)$ & $(5,6,7,8,8)$ & $(3,8,9,9,9)(\mathrm{NA}=1)$ & $(3,6,7,8,9)(N A=2)$ \\
\hline Industry sponsorship (yes) & $4(100 \%)$ & $8(100 \%)$ & $10(100 \%)$ & $8(100 \%)(N A=1)$ & $30(100 \%)(N A=1)$ \\
\hline Exclusion of placebo responders (yes) & $4(100 \%)$ & $5(63 \%)$ & $10(100 \%)$ & $6(75 \%)(N A=1)$ & $25(83 \%)(\mathrm{NA}=1)$ \\
\hline Patient type & & & $(\mathrm{NA}=1)$ & $(\mathrm{NA}=1)$ & $(N A=2)$ \\
\hline Inpatients & $1(25 \%)$ & $2(25 \%)$ & $1(11 \%)$ & $1(12.5 \%)$ & $5(17 \%)$ \\
\hline Outpatients & $3(75 \%)$ & $5(63 \%)$ & $7(78 \%)$ & $6(75 \%)$ & $21(73 \%)$ \\
\hline Outpatients in primary care & $0(0 \%)$ & $1(12 \%)$ & $1(11 \%)$ & $1(12.5 \%)$ & $3(10 \%)$ \\
\hline \multicolumn{6}{|l|}{ Scale used } \\
\hline HDRS & $4(100 \%)$ & $5(63 \%)$ & $4(40 \%)$ & $8(89 \%)$ & $23(74 \%)$ \\
\hline MADRS & $0(0 \%)$ & $3(37 \%)$ & $6(60 \%)$ & $1(11 \%)$ & $8(26 \%)$ \\
\hline \multicolumn{6}{|l|}{ Type of analysis } \\
\hline ITT with LOCF & $4(100 \%)$ & $8(100 \%)$ & $10(100 \%)$ & $8(89 \%)$ & $30(97 \%)$ \\
\hline Mixed model & $0(0 \%)$ & $0(0 \%)$ & $0(0 \%)$ & $1(11 \%)$ & $1(3 \%)$ \\
\hline Initial severity (HDRS score $\geq 25$ ) & $3(75 \%)$ & $5(63 \%)$ & $6(60 \%)$ & $3(37.5 \%)(N A=1)$ & $17(57 \%)(N A=1)$ \\
\hline
\end{tabular}

$\mathrm{n}$, number of studies. Results are presented in (minimum, first quartile, median, third quartile, maximum) for quantitative data except for $V$ vs $F$ vs $P$ where all the data are given. Qualitative data are presented as a number (percentage). NA represents the number of studies where data were unavailable.

$\checkmark$ vs F vs P: studies comparing venlafaxine to fluoxetine to placebo.

$\checkmark$ vs F: studies comparing venlafaxine to fluoxetine.

$\checkmark$ vs $P$ : studies comparing venlafaxine to placebo.

F vs P: studies comparing fluoxetine to placebo.

HDRS, Hamilton Depression Rating Scale; MADRS, Montgomery-Åsberg Depression Rating Scale.

was more efficacious than fluoxetine. Results of the random effect meta-analysis were coherent and are presented in the web-appendix (Additional file 1: Table S4).

\section{Risk of bias across studies}

Six funnel plots were drawn (three for response and three for remission for each head-to-head direct metaanalysis, Figure 4). The funnel plots of the meta-analysis comparing venlafaxine to fluoxetine showed some asymmetry $(P<0.03$ for response and $P<0.01$ for remission). The asymmetry was less striking for funnel plots of meta-analyses for both active treatments versus placebo.

\section{Discussion}

\section{Summary of evidence}

The three placebos considered were not significantly different in terms of response or remission. Antidepressant agents were significantly more efficacious than placebos, and venlafaxine was more efficacious than fluoxetine. This was coherent with previous meta-analyses [7,24]. Thus, venlafaxine placebo appears as a "me-too" placebo without greater benefit in relation to fluoxetine placebo and/or fluoxetine and venlafaxine placebo.

Since, obviously, no direct evidence of well-powered, randomized, direct-comparisons exist, indirect comparisons were the only option for comparing the three different placebos. Such indirect evidence is not totally the same as direct evidence and in some cases it has been shown that indirect comparisons led to conflicting results as compared with direct evidence. Such a paradox has been recently shown concerning antidepressants in a recent paper comparing citalopram with its "me-too" escitalopram which found an inconsistency between direct evidence (showing a superiority of escitalopram) and indirect evidence (which did not find any significant difference) [25]. Additionally, Song et al. have suggested that in some cases indirect evidence is less biased than direct evidence [26]. Moreover, the validity of indirect comparison is dependent on the distribution of relative treatment effect modifiers across different comparisons [27]. In our study, initial severity (HDRS score $\geq 25$ ) could be an important effect modifier [4,5] but despite slight variations, its distribution seemed well-balanced across the different direct comparisons (that is, there was no systematic difference in its distribution between the different direct comparisons).

Nevertheless, results of our indirect comparisons were consistent with the implicit conception that if the three placebos (all made of the same sucrose) were compared in a double blind randomized trial, no difference would 


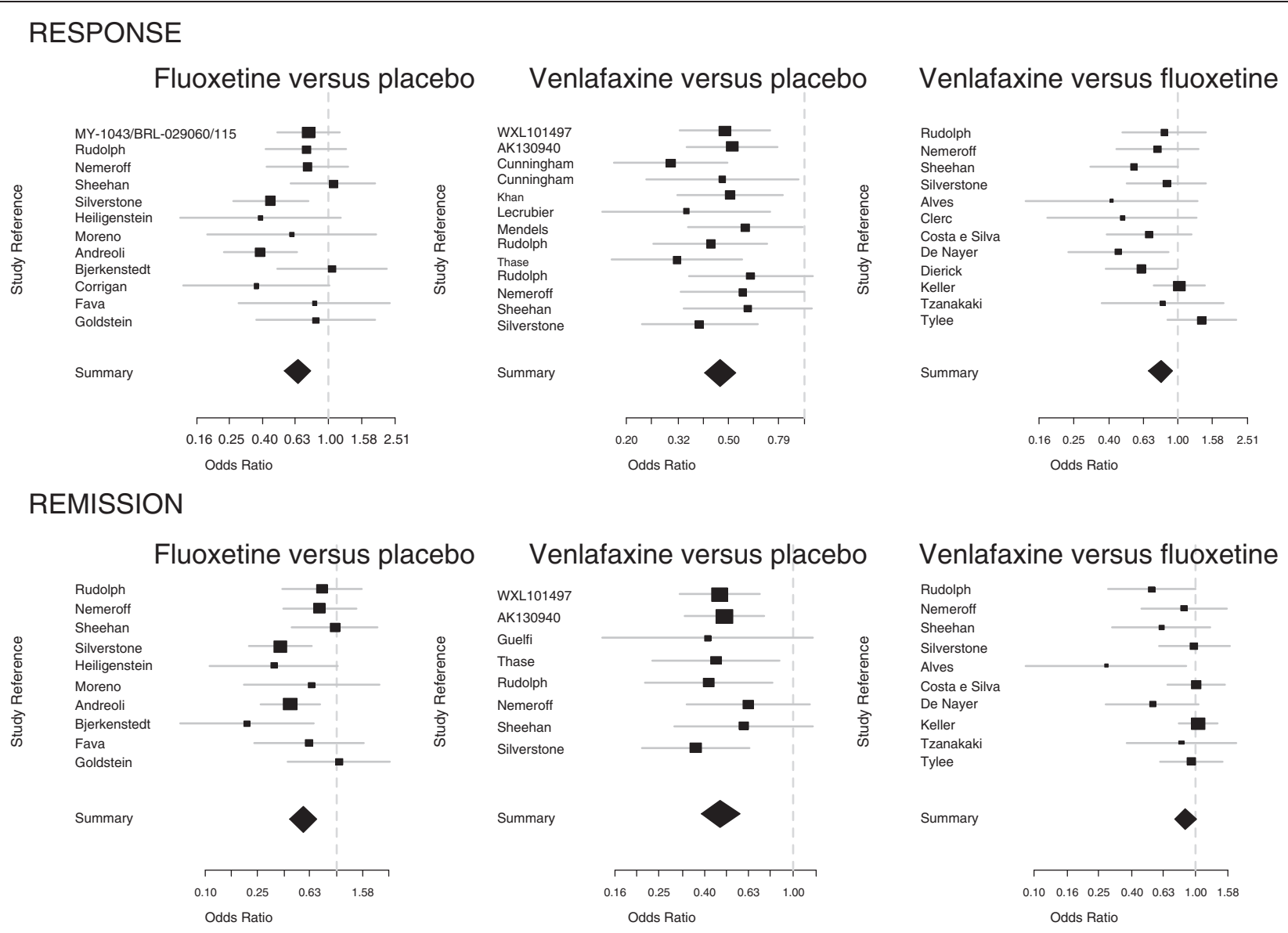

Figure 2 Forest plots. Forest plots present head-to-head meta-analyses using fixed-effects model for response and remission comparing 1) placebo vs fluoxetine, 2) placebo vs venlafaxine and 3) fluoxetine vs venlafaxine.

be observed. These results are good news indeed: 1) for the supporters of placebo because they conclude that the choice of the placebo is not really important, 2) for the supporters of antidepressants because antidepressants prove superior to placebos, and 3) for supporters of rationality because a validated method has not led to invalid conclusions, that is, we have managed to conclude that "sucrose equals sucrose" in the treatment of major depressive disorder, which is wholly reassuring. Yet significant limitations question these findings.

\section{Limitations}

The quality of a meta-analysis is linked to the quality of the individual studies included [28]. In this respect, the National Institute for Health and Clinical Excellence (NICE) guideline on the treatment and management of depression in adults [29] advises caution in the application of results from RCTs and meta-analyses in routine practice. In particular, our model is based on the postulate that all placebo responders should be antidepressant responders (additive model). This key assumption has never been proved to be true. Indeed, antidepressant response and placebo response could be independent or at least substantially overlapping phenomena (nonadditive model), with four different types of patients: 1) placebo-only responders, 2) treatment-only responders, 3) placebo and treatment responders, and 4) never responders [30]. Moreover, the classic logic of the randomized controlled trial casts the placebo effect as a negative foil for measuring therapeutic efficacy and a large amount of important information concerning placebo is not reported in these studies, such as the appearance of the medication (size, shape and color of the pills) [31].

As well, the indirect comparison may have had low statistical power. Even if no trend toward statistical significance was observed within the indirect comparisons of placebos, insignificant $P$-values never tell much about equivalence [32].

Above all, a publication bias and a selective outcome reporting bias might account for some of the effects we observed. The funnel plot for comparison between venlafaxine and fluoxetine shows some asymmetry in favor of venlafaxine. As "true heterogeneity" could not explain this result, and since studies on antidepressants generate 


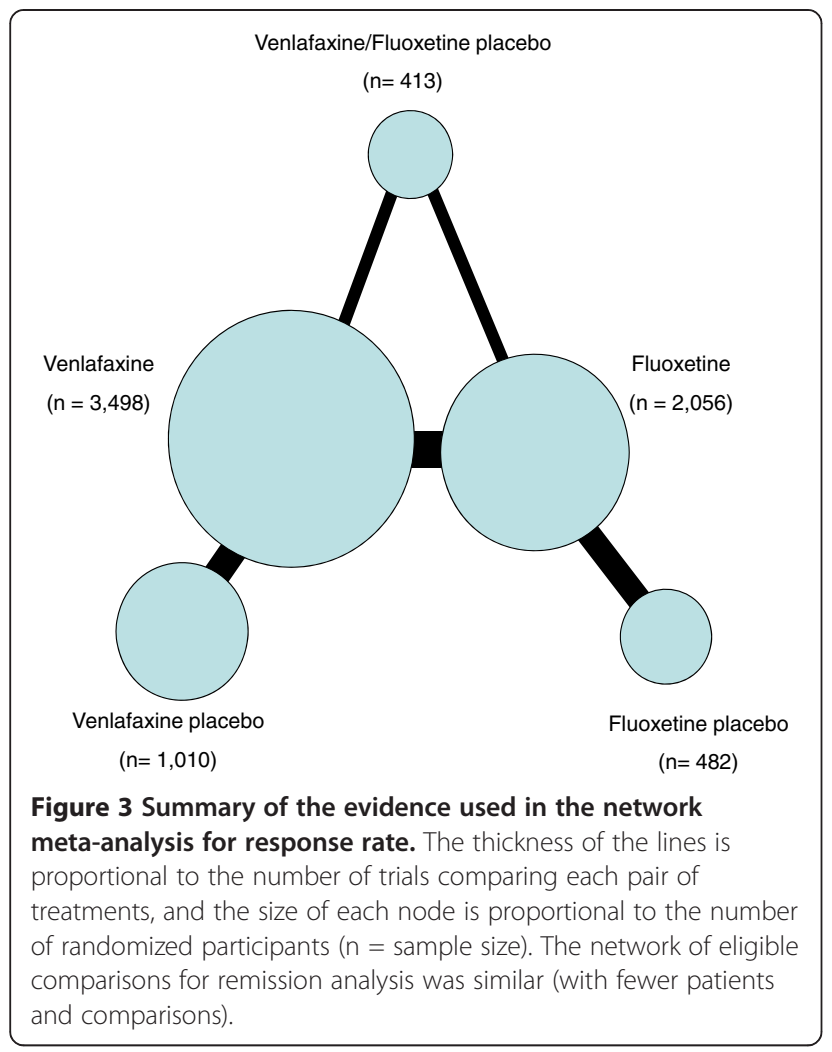

substantial conflicts of interest (these drugs generate vast sales revenues), the result is very much open to suspicion of reporting bias [17]. Concerning comparisons between active antidepressants and placebo, no evidence was found for a publication bias, but as statistical tests for asymmetry typically have low power [17], this bias cannot be excluded. The bias is well known [33] and, for example, it has led to considering reboxetine as a serious antidepressant agent, whereas it is probably ineffective and potentially harmful [34]. It has been recently demonstrated that the selective reporting of studies in network meta-analysis of antidepressants biases estimates of relative treatment efficacy [35].

Various barriers were encountered in our meta-analytic quest for exhaustiveness: many trials were carried out in China and published in Chinese journals. Nevertheless, the quality of many of these studies could be expected to be poor [36], and excluding these trials, although it means a loss of randomized evidence, thus avoids other major biases.

Moreover - and this is probably the main problem - antidepressant research is completely controlled by the pharmaceutical industry [37]: 1) the firms that promoted some of the trials we identified refused to communicate results from these studies; 2) in this meta-analysis all the studies were sponsored by the pharmaceutical industry. Such studies have been shown to be more likely to demonstrate positive effects for the sponsor's drug than independent studies [38].

\section{Perspectives}

Thus, in view of these limitations, a reasonable measure of skepticism should discourage hasty conclusions. They illustrate the fact that every scientific result is uncertain and that it is difficult to be sure of an individual study conclusion, even if it explored something as patently obvious as "sucrose = sucrose", however rigorous the method. Nevertheless, although published research findings can be erroneous [39], Science often generates representations that leave no room for skepticism. This is probably the most insidious pitfall in Evidence-Based Medicine; it does not concern the findings of Science, it concerns academics' understanding of Science (the knowledge-producing activity). The present-day context of medicalization of modern society [40] implicitly dictates that scientific results should have the status of Truth. Concerning major depressive disorder, although it is likely to be untrue, clinicians and a great number of patients [41] strongly believe that antidepressant drugs target a specific biological state that produces depression [42] and the pleasing serotonin hypothesis is often taken as gospel [43].

As for the study by Cipriani et al., it was disputed with similar arguments to those set out in the present study [37,44-47] and its results were not replicated by Gartlehner et al. [48]. Moreover, whereas in our study insignificant $P$-values do not tell much about equivalence, in over-powered studies, like Cipriani's study, statistically significant differences never tell much about clinically significant differences [32]. It is nevertheless mentioned in the NICE guideline [29], with some kind of double

Table 2 Results of the network meta-analyses

\begin{tabular}{|c|c|c|c|c|c|c|c|c|c|}
\hline & FLUp & 1.03 & [0.75 to 1.41$]$ & 0.88 & [0.63 to 1.25$]$ & 0.58 & [0.46 to 0.74 ] & 0.46 & [0.35 to 0.60$]$ \\
\hline 0.94 & [0.61 to 1.43$]$ & & ENLAFp & 0.86 & [0.64 to 1.16$]$ & 0.57 & [0.46 to 0.70$]$ & 0.45 & [0.37 to 0.52 ] \\
\hline 0.91 & [0.59 to 1.39 ] & 0.97 & [0.66 to 1.42$]$ & \multicolumn{2}{|c|}{ FLU/VENLAFp } & $\underline{0.66}$ & [0.51 to 0.84 ] & 0.51 & [0.40 to 0.66 ] \\
\hline 0.51 & $\underline{[0.37 \text { to } 0.71]}$ & $\underline{0.55}$ & [0.41 to 0.73$]$ & 0.57 & $\underline{[0.42 \text { to } 0.76]}$ & & FLU & 0.79 & [0.68 to 0.91$]$ \\
\hline 0.44 & [0.31 to 0.63$]$ & 0.47 & [0.37 to 0.61$]$ & 0.49 & [0.36 to 0.65$]$ & 0.86 & [0.74 to 1.00$]$ & & VENLAF \\
\hline
\end{tabular}

Results of the network meta-analyses are the Odds ratio (OR) between treatment in the column and treatment in the row with their $95 \%$ confidence interval. For response (in bold), OR higher than one favor the treatment indicated in the row. For remission (not in bold), OR higher than one favor the treatment indicated in the column. To obtain OR for comparisons in the opposite direction, reciprocals should be taken. Significant results are underscored. 


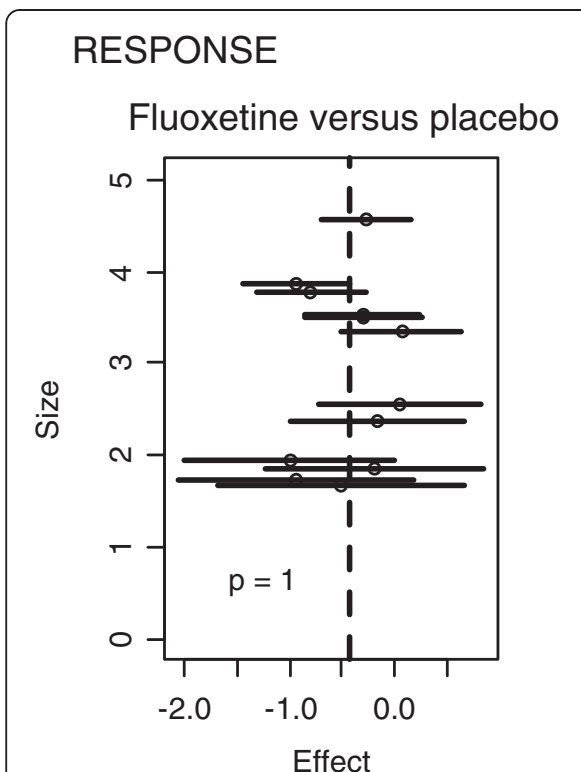

\section{REMISSION}

Fluoxetine versus placebo

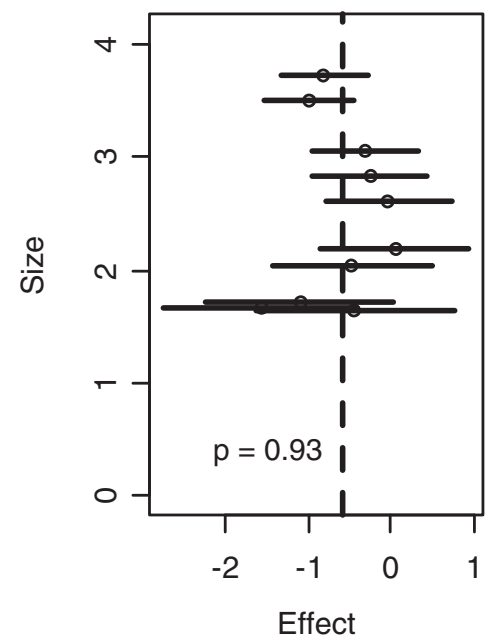

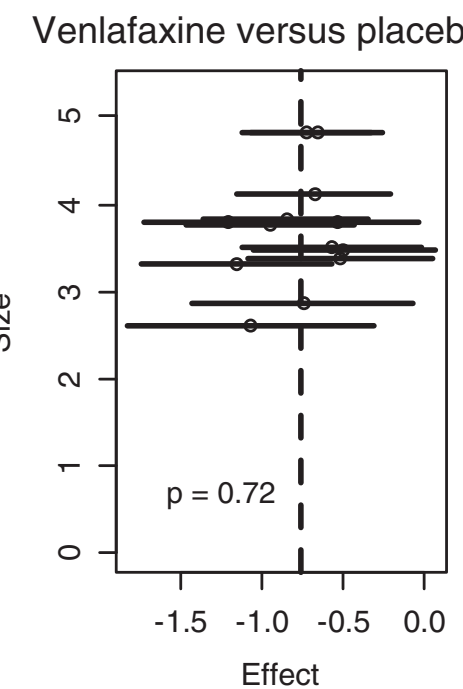

Venlafaxine versus placebo

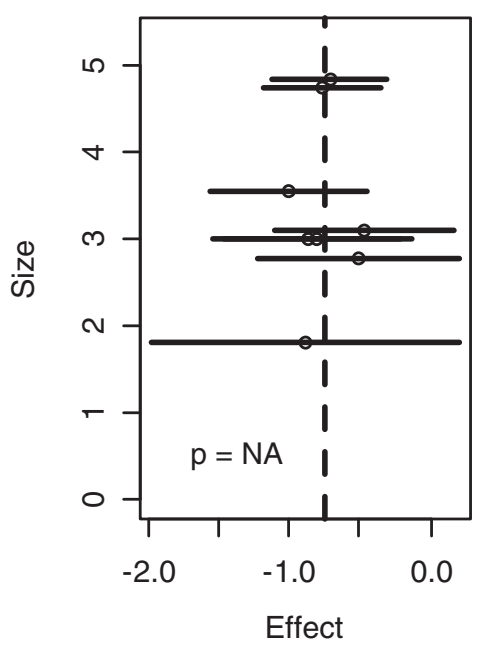

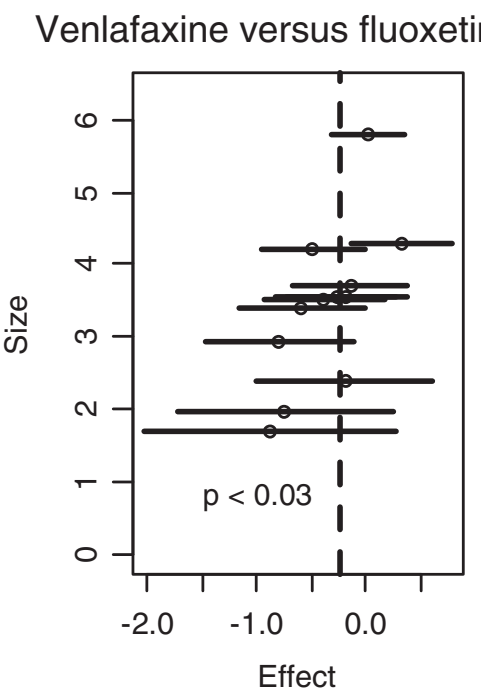

Venlafaxine versus fluoxetine

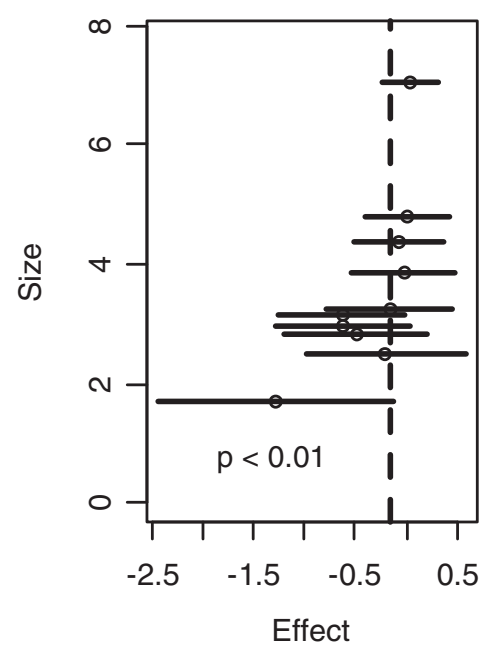

Figure 4 Funnel plots. Six funnel plots were drawn: three for response and three for remission, for each head to head direct meta-analysis. The $P$-value given for each funnel is the result of the rank correlation test of funnel plot asymmetry. NA, test not applicable ( $<10$ studies in the meta-analysis).

bind: qualitatively no recommendations for ranking antidepressants are made, but quantitatively special emphasis is placed on the study (Cipriani's name is cited 23 times versus 3 times for Gartlehner, tables present the results, and so on). What is more, in day-to-day practice, clinicians generally consider that Cipriani's study is solid evidence for choosing antidepressants when treating a patient with newly diagnosed depression [49]. Uncertainty is sometimes acknowledged theoretically, but not in clinical practice.

This epistemological position translates into the wellknown anthropological observation that the hopes and expectations of the physician are just as crucial as those of the patient in the healing process [50]. This suggests that while the opposite may not be true, the best placebos to treat Major Depressive disorder could be antidepressants because they are believed to be effective, which is probably an important determinant in the placebo effect [51].

Nonetheless, we cannot simply assume that, because patients appear to improve on placebos in the short term, as we observe in randomized controlled trials, placebos have demonstrated the required cost-benefit balance. When one uses a treatment that relies on expectation, one 
must also be careful as to its possible harmful consequences, which could be linked with the corollary, disappointment. But here again this is an unsolved central question in Evidence Based Medicine.

\section{Conclusion}

\section{Implications for research}

As in pre-scientific medicine, modern physicians need to believe in the effectiveness of their techniques, and current medical literature, with its strengths and also its limitations, appears as the sophisticated way to generate such beliefs. It also raises the ethical issue of the dissemination of scientific evidence, for example, when editors permit reprints for the pharmaceutical industry intended to present results to doctors via a more commercial than epistemological approach, as was the case for the Cipriani study [52].

\section{Clinical implications}

We did not find any superiority of one placebo over the other. However, a critical approach to our results prevents any firm conclusion on this apparently obvious result. This result should remind clinicians to step back to take a more objective view when interpreting a scientific result, keeping in mind that Science can never be actually sure that "sucrose $=$ sucrose" in the treatment of major depressive disorder. It is of crucial importance for their practice, far more so than ranking antidepressant efficacy.

\section{Additional file}

Additional file 1: Table S1. Studies included and their quality assessment according to the standardized critical appraisal instrument from the Joanna Briggs Institute. Table S2. Head to head meta-analyses (using fixed-effects model (Mantel-Haenszel $=\mathrm{MH}$ ) and random-effects (DerSimonian and Laird = DSL)) of response and remission between 1) placebo vs fluoxetine, 2) placebo vs venlafaxine and 3) fluoxetine vs venlafaxine. Table S3. Akaïke's Information Criterion (AIC) for fixed effect and random effects meta-analyses of response and remission. Table S4. Odds ratio (OR) of response and remission between fluoxetine placebo, venlafaxine placebo, fluoxetine/venlafaxine placebo, fluoxetine and venlafaxine. Figure S1. Forest plot presenting head-to-head meta-analyses using random-effects model for response and remission comparing 1) placebo vs fluoxetine, 2) placebo vs venlafaxine and 3) fluoxetine vs venlafaxine.

\footnotetext{
Abbreviations

AIC: Akaïke's information criterion; DSM III: Diagnostic and statistical manual of mental disorders, $3^{\text {rd }}$ edition; DSM III-R: Diagnostic and statistical manual of mental disorders, $3^{\text {rd }}$ edition, text revised; DSM IV: Diagnostic and statistical manual of mental disorders, $4^{\text {th }}$ edition; DSM IV-R: Diagnostic and statistical manual of mental disorders, $4^{\text {th }}$ edition, text revised; EMEA: European medicines agency; FDA: Food and drug administration; FLUNENLAFp: Venlafaxine/fluoxetine placebo; FLUp: Fluoxetine placebo; HDRS: Hamilton depression rating scale; ICD 10: International classification of diseases, 10th Revision; LOCF: Last observation carried forward; MADRS: Montgomery-Åsberg depression rating scale; NICE: National institute for health and clinical excellence; OR: Odds ratio; PRISMA: Preferred reporting items for systematic reviews and meta-analyses; RCTs: Randomized controlled trials; VENLAFp: Venlafaxine placebo.
}

\section{Competing interests}

There are no conflicts of interest regarding this paper. All authors have completed the Unified Competing Interest form at http://www.icmje.org/ coi_disclosure.pdf (available on request from the corresponding author) and declare that no authors have support from any company for the submitted work. NF has relationships (board membership or Travel/accommodations expenses covered/reimbursed) with Servier, BMS, Lundbeck and Janssen who might have an interest in the work submitted in the previous three years. MB has relationships (consultancy and travel/accommodations expenses covered/reimbursed) with Janssen, BMS, Otsuka, Lundbeck, Lilly, Servier, Astra Zeneca, Medtronics, Syneïka and has received grants for research from Medtronic, Lilly and Astra Zeneca in the previous three years. $\mathrm{CP}, \mathrm{RJM}$ and MAS have no relationships with any company that might have an interest in the submitted work in the previous three years. FB has relationships (board membership or consultancy or payment for manuscript preparation or Travel/accommodations expenses covered/reimbursed) with Sanofi-Aventis, Servier, Pierre-Fabre, MSD, Lilly, Janssen-Cilag, Otsuka, Lundbeck, Genzime, Roche and BMS who might have an interest in the work submitted in the previous three years. NF, CP, MAS, RJM, FB and their spouses, partners or children have no financial relationships that may be relevant to the submitted work; MB's spouse is an employee of Janssen. None of the authors has any non-financial interests that may be relevant to the submitted work.

\section{Authors' contributions}

FN conceived and designed the experiments, performed the experiments, analyzed the data and wrote the paper. FN had full access to all of the data in the study and takes responsibility for the integrity of the data and the accuracy of the data analysis. BM, PC and JMR revised the paper critically for important intellectual content. ASM performed the experiments. BF participated in the design of the experiments, performed the experiments and revised the paper critically for important intellectual content. All authors read and approved the final manuscript.

\section{Acknowledgments}

We thank all the authors of the different studies who cooperated, Guillemette Utard for her advice on bibliographic research, Lionel Riou França, Hervé Maisonneuve and Nicolas Naudet for their very interesting comments, Claudine Naudet, and Angela Swaine Verdier for revising the English.

ASM is funded by the Conseil General d'lle de France (PICRI). This paper was supported by the Institut National de la Santé et de la Recherche Médicale (INSERM). The sponsor had no role concerning the design and conduct of the study; collection, management, analysis, and interpretation of the data; and preparation, review, or approval of the manuscript.

\section{Author details}

'INSERM U669, Maison de Solenn, 97 Boulevard de Port Royal, 75679 Paris Cedex 14, France. ${ }^{2}$ Université de Rennes 1, EA-4712 Behavior and Basal Ganglia Unit, Rennes, France. ${ }^{3}$ Centre Hospitalier Guillaume Régnier, Service Hospitalo-Universitaire de Psychiatrie, Rennes, France. ${ }^{4}$ Department of Forensic Medicine and Pathology, University Hospital R. Poincaré (AP-HP, UVSQ), Garches, France. ${ }^{5}$ Laboratory of Medical Ethics, University of Paris 5 , 45 St Pères Street, 75006 Paris, France. ${ }^{6}$ Centre d'Investigation Clinique CIC-P INSERM 0203, Hôpital de Pontchaillou, Centre Hospitalier Universitaire de Rennes et Université de Rennes 1, Rennes, France. ${ }^{7}$ Laboratoire de Pharmacologie Expérimentale et Clinique, Faculté de Médecine, CS34317, 2 Avenue du Pr Léon Bernard, 35043 Rennes, France. ${ }^{8}$ Université Paris-Sud and Université Paris Descartes, UMR-S0669, Paris, France. ${ }^{9}$ AP-HP, Hôpital Paul Brousse, Département de santé publique, Villejuif, France.

Received: 7 February 2013 Accepted: 4 October 2013

Published: 25 October 2013

\section{References}

1. Benedetti F: Placebo Effects: Understanding the Mechanisms in Health and Disease. Oxford, UK: Oxford University Press; 2009.

2. Kaptchuk TJ, Kerr CE, Zanger A: Placebo controls, exorcisms and the devil. Lancet 2009, 374:1234. 
3. Ioannidis JP: Effectiveness of antidepressants: an evidence myth constructed from a thousand randomized trials? Philos Ethics Humanit Med 2008, 3:14.

4. Kirsch I, Deacon BJ, Huedo-Medina TB, Scoboria A, Moore TJ, Johnson BT: Initial severity and antidepressant benefits: a meta-analysis of data submitted to the Food and Drug Administration. PLoS Med 2008, 5:e45

5. Fournier JC, DeRubeis RJ, Hollon SD, Dimidjian S, Amsterdam JD, Shelton RC, Fawcett J: Antidepressant drug effects and depression severity: a patient-level meta-analysis. JAMA 2010, 303:47-53.

6. Moncrieff J: The Myth of the Chemical Cure: A Critique of Psychiatric Drug Treatment. Revised Edition. Basingstoke, UK: Palgrave Macmillan; 2009.

7. Cipriani A, Furukawa TA, Salanti G, Geddes JR, Higgins JP, Churchill R, Watanabe N, Nakagawa A, Omori IM, McGuire H, Tansella M, Barbui C: Comparative efficacy and acceptability of 12 new-generation antidepressants: a multiple-treatments meta-analysis. Lancet 2009, 373:746-758.

8. Hamilton M: A rating scale for depression. J Neurol Neurosurg Psychiatry 1960, 23:56-62.

9. Montgomery SA, Asberg M: A new depression scale designed to be sensitive to change. Br J Psychiatry 1979, 134:382-389.

10. Naudet F, Maria AS, Falissard B: Antidepressant response in majo depressive disorder: a meta-regression comparison of randomized controlled trials and observational studies. PLoS One 2011, 6:e20811.

11. Joanabrigss Institute: Protocols and work in progress. [http://joannabriggs.org]

12. In Cochrane Handbook for Systematic Reviews of Interventions. Version 5.1.0. Updated March 2011. Edited by Higgins JP, Green S. [http://www. cochrane-handbook.org/]

13. Hedges L, Olkin I: Statistical Methods for Meta-analysis. New York, NY: Academic Press; 1985.

14. Glenny AM, Altman DG, Song F, Sakarovitch C, Deeks JJ, D'Amico R, Bradburn M, Eastwood AJ: Indirect comparisons of competing interventions. Health Technol Assess 2005, 9:1-134.

15. Jansen JP, Fleurence $R$, Devine $B$, Itzler $R$, Barrett A, Hawkins N, Lee $K$ Boersma C, Annemans L, Cappelleri JC: Interpreting indirect treatment comparisons and network meta-analysis for health-care decision making: report of the ISPOR Task Force on Indirect Treatment Comparisons Good Research Practices: part 1. Value Health 2011, 14:417-428.

16. Hoaglin DC, Hawkins N, Jansen JP, Scott DA, Itzler R, Cappelleri JC, Boersma C, Thompson D, Larholt KM, Diaz M, Barrett A: Conducting indirecttreatment-comparison and network-meta-analysis studies: report of the ISPOR Task Force on Indirect Treatment Comparisons Good Research Practices: part 2. Value Health 2011, 14:429-437.

17. Sterne JA, Sutton AJ, loannidis JP, Terrin N, Jones DR, Lau J, Carpenter J, Rucker G, Harbord RM, Schmid CH, Tetzlaff J, Deeks JJ, Peters J, Macaskill P, Schwarzer G, Duval S, Altman DG, Moher D, Higgins JP: Recommendations for examining and interpreting funnel plot asymmetry in meta-analyses of randomised controlled trials. BMJ 2011, 343:d4002.

18. R Development Core Team: R: A Language and Environment for Statistical Computing. Vienna, Austria: R Foundation for Statistical Computing; 2009.

19. Schwarzer G: meta: Meta-Analysis with R, R Package version 11-8. Vienna: The Comprehensive R Archive Network; 2010.

20. Lumley T: rmeta: Meta-analysis, $R$ package version 216. Vienna: The Comprehensive R Archive Network; 2009.

21. Maechler DBM: Ime4: Linear Mixed-effects Models Using S4 Classes, R Package Version 0999375-32. Vienna: The Comprehensive R Archive Network; 2009.

22. Liberati A, Altman DG, Tetzlaff J, Mulrow C, Gotzsche PC, loannidis JP, Clarke M, Devereaux PJ, Kleijnen J, Moher D: The PRISMA statement for reporting systematic reviews and meta-analyses of studies that evaluate health care interventions: explanation and elaboration. PLoS Med 2009, 6:e1000100

23. Higgins JP, Thompson SG, Deeks JJ, Altman DG: Measuring inconsistency in meta-analyses. BMJ 2003, 327:557-560

24. Bauer $M$, Tharmanathan $P$, Volz HP, Moeller HJ, Freemantle $\mathrm{N}$ : The effect of venlafaxine compared with other antidepressants and placebo in the treatment of major depression: a meta-analysis. Eur Arch Psychiatry Clin Neurosci 2009, 259:172-185.

25. Alkhafaji AA, Trinquart L, Baron G, Desvarieux M, Ravaud P: Impact of evergreening on patients and health insurance: a meta analysis and reimbursement cost analysis of citalopram/escitalopram antidepressants. BMC Med 2012, 10:142
26. Song F, Harvey I, Lilford R: Adjusted indirect comparison may be less biased than direct comparison for evaluating new pharmaceutical interventions. J Clin Epidemiol 2008, 61:455-463.

27. Jansen JP, Naci H: Is network meta-analysis as valid as standard pairwise meta-analysis? It all depends on the distribution of effect modifiers. BMC Med 2013, 11:159.

28. Egger M, Smith GD, Sterne JA: Uses and abuses of meta-analysis. Clin Med 2001, 1:478-484

29. National Collaborating Centre for Mental Health (UK): Depression: The Treatment and Management of Depression in Adults. (Updated Edition). NICE Clinical Guidelines, No. 90. Leicester, UK: British Psychological Society; 2010.

30. Kirsch I: Are drug and placebo effects in depression additive? Biol Psychiatry 2000, 47:733-735.

31. Greene JA, Kesselheim AS: Why do the same drugs look different? Pills, trade dress, and public health. N Engl J Med 2011, 365:83-89.

32. Altman DG, Bland JM: Absence of evidence is not evidence of absence. BMJ 1995, 311:485.

33. Turner EH, Matthews AM, Linardatos E, Tell RA, Rosenthal R: Selective publication of antidepressant trials and its influence on apparent efficacy. N Engl J Med 2008, 358:252-260.

34. Eyding $D$, Lelgemann $M$, Grouven $U$, Harter $M$, Kromp $M$, Kaiser $T$, Kerekes MF, Gerken M, Wieseler B: Reboxetine for acute treatment of major depression: systematic review and meta-analysis of published and unpublished placebo and selective serotonin reuptake inhibitor controlled trials. BMJ 2010, 341:c4737

35. Trinquart $L$, Abbe $A$, Ravaud P: Impact of reporting bias in network meta-analysis of antidepressant placebo-controlled trials. PLoS One 2012 7:e35219.

36. Wu T, Li Y, Bian Z, Liu G, Moher D: Randomized trials published in some Chinese journals: how many are randomized? Trials 2009, 10:46.

37. Ioannidis JP: Ranking antidepressants. Lancet 2009, 373:1759-1760. author reply 1761-1762.

38. Lexchin J, Bero LA, Djulbegovic B, Clark O: Pharmaceutical industry sponsorship and research outcome and quality: systematic review. BMJ 2003, 326:1167-1170.

39. loannidis JP: Why most published research findings are false. PLOS Med 2005, 2:e124.

40. Rose N: Beyond medicalisation. Lancet 2007, 369:700-702.

41. Pescosolido BA, Martin JK, Long JS, Medina TR, Phelan JC, Link BG: "A disease like any other"? A decade of change in public reactions to schizophrenia, depression, and alcohol dependence. Am J Psychiatry 2010, 167:1321-1330

42. Moncrieff J, Cohen D: Do antidepressants cure or create abnormal brain states? PLoS Med 2006, 3:e240.

43. Lacasse JR, Leo J: Serotonin and depression: a disconnect between the advertisements and the scientific literature. PLoS Med 2005, 2:e392.

44. Jefferson T: Ranking antidepressants. Lancet 2009, 373:1759. author reply 1761-1762.

45. Schwan S, Hallberg P: Ranking antidepressants. Lancet 2009, 373:1761. author reply 1761-1762.

46. Seyringer ME, Kasper S: Ranking antidepressants. Lancet 2009, 373:1760-1761 author reply 1761-1762.

47. Turner E, Moreno SG, Sutton AJ: Ranking antidepressants. Lancet 2009, 373:1760. author reply 1761-1762.

48. Gartlehner G, Gaynes BN, Hansen RA, Thieda P, DeVeaugh-Geiss A, Krebs EE, Moore CG, Morgan L, Lohr KN: Comparative benefits and harms of second-generation antidepressants: background paper for the American College of Physicians. Ann Intern Med 2008, 149:734-750.

49. Patrick G, Combs G, Gavagan T: Initiating antidepressant therapy? Try these 2 drugs first. J Fam Pract 2009, 58:365-369.

50. Lakoff $A$ : The mousetrap: managing the placebo effect in antidepressant trials. Mol Interv 2002, 2:72-76.

51. Thomas KB: General practice consultations: is there any point in being positive? Br Med J (Clin Res Ed) 1987, 294:1200-1202.

52. Handel AE, Patel SV, Pakpoor J, Ebers GC, Goldacre B, Ramagopalan SV: High reprint orders in medical journals and pharmaceutical industry funding: case-control study. BMJ 2012, 344:e4212

doi:10.1186/1741-7015-11-230

Cite this article as: Naudet et al: Which placebo to cure depression? A thought-provoking network meta-analysis. BMC Medicine 2013 11:230. 